\title{
Challenges and opportunities of the COVID-19 pandemic for perinatal mental health care: a mixed-methods study of mental health care staff
}

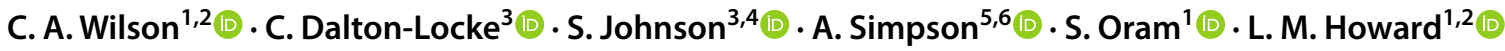

Received: 8 September 2020 / Accepted: 20 January 2021 / Published online: 8 April 2021

(c) The Author(s) 2021

\begin{abstract}
The aim of this study was to explore staff perceptions of the impact of the COVID-19 pandemic on mental health service delivery and outcomes for women who were pregnant or in the first year after birth ('perinatal' women). Secondary analysis was undertaken of an online mixed-methods survey open to all mental health care staff in the UK involving 363 staff working with women in the perinatal period. Staff perceived the mental health of perinatal women to be particularly vulnerable to the impact of stressors associated with the pandemic such as social isolation (rated by $79.3 \%$ as relevant or extremely relevant; 288/363) and domestic violence and abuse $(53.3 \% ; 192 / 360)$. As a result of changes to mental health and other health and social care services, staff reported feeling less able to assess women, particularly their relationship with their baby $(43.3 \%$; 90/208), and to mobilise safeguarding procedures $(29.4 \%$; 62/211). While $42 \%$ of staff reported that some women engaged poorly with virtual appointments, they also found flexible remote consulting to be beneficial for some women and helped time management due to reductions in travel time. Delivery of perinatal care needs to be tailored to women's needs; virtual appointments are perceived not to be appropriate for assessments but may be helpful for some women in subsequent interactions. Safeguarding and other risk assessment procedures must remain robust in spite of modifications made to service delivery during pandemics.
\end{abstract}

Keywords Perinatal $\cdot$ Mental health $\cdot$ COVID-19 $\cdot$ Coronavirus $\cdot$ Pandemic

What is already known on this subject?

There is some emerging evidence that the current COVID-19 pandemic is exerting a significant burden on perinatal mental health.

What does this study add?

Mental health and social care services must urgently evaluate how best to assess and support perinatal mental health during pandemics.

C. A. Wilson

claire.1.wilson@kcl.ac.ukAuthors and Affiliations

1 Section of Women's Mental Health, Institute of Psychiatry, Psychology and Neuroscience, King's College London, London, UK

2 South London and Maudsley NHS Foundation Trust, London, UK

3 NIHR Mental Health Policy Research Unit, Division of Psychiatry, University College London, London, UK

4 Camden and Islington NHS Foundation Trust, London, UK

5 Health Service and Population Research, Institute of Psychiatry, Psychology and Neuroscience, King's College London, London, UK

6 NIHR Mental Health Policy Research Unit, Institute of Psychiatry, Psychology and Neuroscience, King's College London, London, UK

\section{Introduction}

Since the advent of the global COVID-19 pandemic in March 2020, numerous concerns regarding its impact on public mental health have been raised. These include the impact of lockdown and social distancing measures, bereavement and a range of other socio-economic stressors (Adhanom Ghebreyesus 2020). Surveys of adults from around the world have reported increased symptoms of anxiety and depression, with many finding women to be at an increased risk (González-Sanguino et al. 2020; Hyland et al. 2020; Pierce et al. 2020). There are also concerns about the impact of the pandemic on those with existing mental illness (Sheridan Rains et al. 2020). Those with severe mental illness are at increased risk of COVID-19 infection due to higher rates of comorbidities such as physical ill health and socio-economic adversities. They are also more vulnerable to the indirect effects of the pandemic such as reduced service provision and social isolation, which may precipitate relapse (Moreno et al. 2020).

Perinatal mental health services in the UK support women during pregnancy and up to 1 year postpartum with 
a range of mental illnesses, including schizophrenia and bipolar disorder, and the more common mental disorders of anxiety and depression. Pre-pandemic, mental illness affected at least one in five women in the perinatal period and is associated with adverse maternal and child outcomes (Ayers and Shakespeare 2015; Stein et al. 2014). Several factors associated with the pandemic may disproportionately affect this perinatal population already vulnerable to mental ill health. During the early phase of the pandemic, women in the UK were advised to follow stricter social distancing than the general population (UK Government 2020), leading to reduced contact with friends, family and health and social care services (Caparros-Gonzalez and Alderdice 2020). Another factor associated with mental ill health which has increased during the pandemic is violence, abuse and exploitation (Usher et al. 2020). The perinatal period is a time of increased risk for domestic violence and abuse (DVA) and it is associated with adverse maternal and child outcomes (Oram et al. 2017).

There remains limited understanding of the impact of the pandemic on care provided for those with mental illness, including in the perinatal period (The Lancet Psychiatry 2020). However, there are reports of early discharges from inpatient psychiatric units and reduced community service provision, including fewer face-to-face meetings (Moreno et al. 2020). The aim of this study was to explore, among those working in mental health services in the UK, the impact of the pandemic on the mental health care provided to women in the perinatal period.

\section{Materials and methods}

From 22 April 2020 to 12 May 2020, the NIHR Mental Health Policy Research Unit (MHPRU) surveyed staff working across services that provide mental health care in the UK, including the National Health Service (NHS), private services, social care and third sector or voluntary services. The development of the online mixed-methods questionnaire has been previously described and was done in close collaboration with service users and clinicians (Johnson et al. 2020). Both structured and open-ended questions explored staff's perceptions of problems faced by service users and carers and challenges to care provision. These core questions were followed by additional questions specific to staff working in particular settings and specialities. Recruitment was via social media, for example Twitter, and relevant professional bodies, with a particular emphasis on recruiting staff from diverse groups. The King's College London Research Ethics Committee approved the study (MRA-19/20-18372).

The sample comprised those in the survey who reported working with pregnant or postnatal women in generic or perinatal specialist services (community teams or inpatient
Mother and Baby Units: MBUs) and who completed at least one question from each of the three main sections of questions open to all respondents described above, as per the approach taken by a previous analysis of the full sample of respondents (all mental health staff) (Johnson et al. 2020).

Quantitative data were analysed using Stata 15 (StataCorp 2007) and are presented as descriptive statistics. Qualitative data from open-ended questions were analysed using inductive semantic thematic analysis (Braun and Clarke 2006). Initial coding of data led to the identification of potential themes, which were checked against coded extracts in a recursive process. For the purposes of providing context for the qualitative analysis, several of the authors are practising psychiatrists, some of whom work in perinatal psychiatry, although none was practising in perinatal services at the time of the pandemic. Peer debriefing was employed between author C.A.W., who conducted the qualitative analysis, and author L.M.H. (Lincoln and Guba 1985).

\section{Results}

\section{Participant characteristics}

Of 2180 respondents who completed at least one question from each of the three main sections open to all respondents (3712 started the survey and 1793 reached the end of it), 363 reported working with perinatal service users in generic or specialist services. This sample of 363 was used for the analysis. Characteristics of the sample are presented in Table 1. The majority $(330 / 363 ; 91.2 \%)$ worked in the NHS and in England (299/363; 82.4\%). Fifty-four of the staff reported working in a hospital inpatient service, 18 of these in MBUs. $41.6 \%(151 / 363)$ identified as a manager or lead clinician in their service.

\section{Difficulties experienced by users of perinatal mental health services}

Staff were asked to rate the relevance, on a five-point scale, of pre-specified problems among service users and carers with whom they were currently in contact. Table 2 displays proportions of staff rating each item as 'very relevant' or 'extremely relevant'; items are ordered by highest proportion first.

Responses to free text questions revealed similar concerns. Staff were asked for which service users they were especially concerned and new mental health problems arising directly from the pandemic. The majority of staff reported being concerned about women in the perinatal period. Concerns and their impacts grouped as themes and how they inter-relate are displayed in Fig. 1. Difficulties related either to the virus itself or environmental adaptations 
Table 1 Characteristics of staff working with perinatal women $N=363$ staff

\begin{tabular}{ll}
\hline Characteristic & $n(\%)$ \\
\hline Gender & \\
Female & $236(65)$ \\
Male & $41(11.3)$ \\
Missing, N/A or prefer not to say & $86(23.7)$ \\
Ethnicity & \\
White & $224(61.7)$ \\
Asian & $19(5.2)$ \\
Mixed or multiple ethnic groups & $10(2.8)$ \\
Black & $8(2.2)$ \\
Other & $1(0.3)$ \\
Missing, N/A or prefer not to say & $101(27.8)$ \\
Profession & \\
Nurse & $117(32.2)$ \\
Psychiatrist & $47(13)$ \\
Clinical or counselling psychologist & $37(10.2)$ \\
Social worker & $22(6.1)$ \\
Occupational therapist & $19(5.2)$ \\
Peer support worker & $9(2.5)$ \\
Manager & $9(2.5)$ \\
Other qualified therapists & $57(15.7)$ \\
Other work & $45(12.4)$ \\
Missing & $1(0.3)$ \\
Sector* & \\
NHS & 330 \\
Voluntary & 255 \\
Social care or other local government \\
Private & 78 \\
Community or user-led organisations & 54 \\
Community mental health team (CMHT) & 26 \\
Crisis service & 5 \\
Inpatient service & 14 \\
Community groups & 11 \\
Residential service & 5 \\
Crisis house & \\
Other & \\
\hline
\end{tabular}

*Staff could report working in multiple settings or sectors

to reduce transmission. A few staff described cases of mental illness in women testing positive for COVID-19, including postpartum psychosis. Other staff reflected on the contribution of the current viral pandemic to the content of delusions arising in the context of new-onset or pre-existing psychosis. Another theme was that of fears of COVID-19 infection: 'Pregnant mothers extremely worried about the risk of catching COVID-19 and the risks to their babies'.

Other themes arose due to broader societal restrictions. Most staff reported concerns about loss of support and subsequent isolation in the context of reduced contact with family, friends and professionals during pregnancy and in the postpartum: 'perinatal period ... difficult time for some, involving some isolation. With the lockdown, many pregnant women were left having remote appointments'. Another closely related theme was concerns about those at an increased risk of domestic violence and abuse (DVA): 'Vulnerable women and children at higher risk of DV' and 'Domestic abuse situations increasing', alongside feelings from staff of powerlessness to intervene, which will be considered further in the section that follows.

Most participants reported increases in depression and anxiety disorders among perinatal women, including worsening or relapse of pre-pandemic disorders or new symptoms since the pandemic's onset. One staff respondent wrote: 'Anxiety and OCD [Obsessive Compulsive Disorder] symptoms related to fear of infection'. Another commented: 'Impacting on mood and increasing risk of harm to self', when reflecting on the impact of social isolation. A few staff noted an increase in substance use: 'increased substance misuse among perinatal women'. Another theme which may have contributed to anxiety and depression in perinatal women was increased uncertainty and lack of control, already prominent during the perinatal period: 'Feelings of being out of control'. Finally, a few staff reflected that women's experiences of having a baby during the pandemic had been very different from what they had expected: 'women due to give birth ... very concerned about their birth experience' and 'Anxiety related to change in birth plans ... Birth partner not being present at birth of baby'. A possible increased risk of birth trauma was noted by some staff: 'Birth trauma due to restrictions in maternity services'. Many staff were particularly concerned about the post-traumatic disorder (PTSD) in perinatal women in the longer term.

\section{Changes to perinatal mental health care}

Staff were also asked about changes made to services during the pandemic. 44.4\% (92/207) of staff working in community teams estimated that referral rates declined by more than $10 \%$. In inpatient services, $54.8 \%$ (17/31) of staff estimated that the monthly admission rate declined by more than $10 \%$.

All respondents were asked to rate the relevance of challenges to current work in the pandemic (since mid-March 2020). These differed somewhat between inpatient and residential settings (hospital inpatient service, crisis houses and residential services) and community settings (CMHTs, crisis teams and community groups). Four challenges rated most frequently as very or extremely relevant in inpatient and residential settings were pressures of supporting colleagues $(31 / 58 ; 53.4 \%)$, adapting to new ways of working $(31 / 58$; $53.4 \%)$, being infected with COVID-19 at work (30/58; 
Table 2 Difficulties rated as very or extremely relevant for mental health service users and their carers identified by mental health staff

\begin{tabular}{|c|c|c|}
\hline & $n / N^{*}$ & $\%$ \\
\hline Lack of access to usual support networks of family and friends & $288 / 363$ & 79.3 \\
\hline Loneliness due to or made worse by social distancing, self-isolation and/or shielding & $275 / 363$ & 75.8 \\
\hline Lack of usual work and activities & $245 / 358$ & 68.4 \\
\hline Worries about getting COVID-19 infection & $240 / 363$ & 66.1 \\
\hline Lack of access to usual support from other services (primary care, social care, voluntary sector) & $232 / 363$ & 63.9 \\
\hline Worries about family getting COVID-19 infection & $227 / 363$ & 62.5 \\
\hline Lack of access to usual support from NHS mental health services & $196 / 362$ & 54.1 \\
\hline Increased risk from abusive domestic relationships & $192 / 360$ & 53.3 \\
\hline Increased difficulties for families/carers & $190 / 360$ & 52.8 \\
\hline Relapse and deterioration in mental health triggered by COVID-19 stresses & $182 / 363$ & 50.1 \\
\hline Increase in reliance on family/family tensions & $164 / 358$ & 45.8 \\
\hline High personal risk of severe consequences of COVID-19 infection (e.g. due to physical health comorbidities) & $155 / 359$ & 43.2 \\
\hline Difficulty engaging with remote appointments by phone or via digital platforms & $153 / 363$ & 42.1 \\
\hline Having to stay at home in poor circumstances or not having a home to go to & $142 / 362$ & 39.2 \\
\hline Difficulty getting food, money or other basic resources & $138 / 362$ & 38.1 \\
\hline Diminished access to physical health care for problems other than COVID-19 & $131 / 362$ & 36.2 \\
\hline Difficulty understanding or following current government requirements on social distancing, self-isolation and/or shielding & $117 / 362$ & 32.3 \\
\hline Effects of COVID-19-related trauma & $113 / 360$ & 31.4 \\
\hline Risk of increased drug and alcohol use or gambling & $108 / 360$ & 30.0 \\
\hline Lack of access to or of equitable provision of physical health care for COVID-19 & $81 / 362$ & 22.4 \\
\hline Lack of access to medication and to processes for administering and monitoring it & $67 / 360$ & 18.6 \\
\hline Loss of liberty and rights due to changes in implementation of mental health legislation & $61 / 360$ & 16.9 \\
\hline $\begin{array}{l}\text { Problems with police or other authorities because of lack of understanding of/ability to stick to current government require- } \\
\text { ments }\end{array}$ & $42 / 362$ & 11.6 \\
\hline
\end{tabular}

*Total respondents for this item

$51.7 \%)$ and putting infection control measures into practice $(28 / 58 ; 48.3 \%)$. The same challenges were identified in staff who reported working in MBUs specifically. In community settings, the most frequently rated as very or extremely relevant were adapting to new ways of working (188/307; $61.2 \%$ ), learning to use new technologies too quickly and/ or without sufficient training and support (147/306; 48\%), responding to additional mental health needs arising from COVID-19 (138/308; 44.8\%) and pressures of supporting colleagues $(123 / 305 ; 40.3 \%)$.

As all staff in the sample reported working with the perinatal population, they were later presented with additional challenges specific to perinatal work. Table 3 displays the proportion of staff rating each item as 'very relevant' or 'extremely relevant'; items are ordered by highest proportion first. Completion rates of these items were just under $60 \%$ of the sample.

Some challenges related to reduced ability to adequately assess and support perinatal women, their babies and their wider families, were echoed in responses to open questions. Themes of changes staff were most concerned about in the perinatal setting and their perceived impact are displayed in Fig. 2. Community staff reported less frequent and fewer face-to-face contacts with women. Staff were concerned about their ability to detect early signs of mental illness when reviewing women remotely. Many were also worried about how to adequately assess and support the mother-infant interaction during the remote consultation by telephone or video: 'unable to [do] vital work to help mums bond with their babies ... only allowed to undertake phone calls not home visits.' In extreme cases where staff are worried about the infant's well-being and have safeguarding concerns, this assessment of interaction is part of the risk formulation as one staff respondent summarised: 'Vulnerable babies being cared for by unwell mothers who are not being identified due to lockdown because they are more isolated and less likely to be picked up by professionals'. Risk assessment may also be challenging in a remote consultation: 'Women with know[n] Domestic Abuse ... doing video consultations so may not be able to be entirely honest about symptoms and risk'. Another barrier to actioning safeguarding concerns identified by some staff was reduced provision from other agencies such as social services. Another theme of reduced community service provision in the postnatal period from other services, such as health visiting, was identified, 
Fig. 1 Concerns from staff about areas of difficulty for perinatal service users and their perceived impacts (themes in boxes)

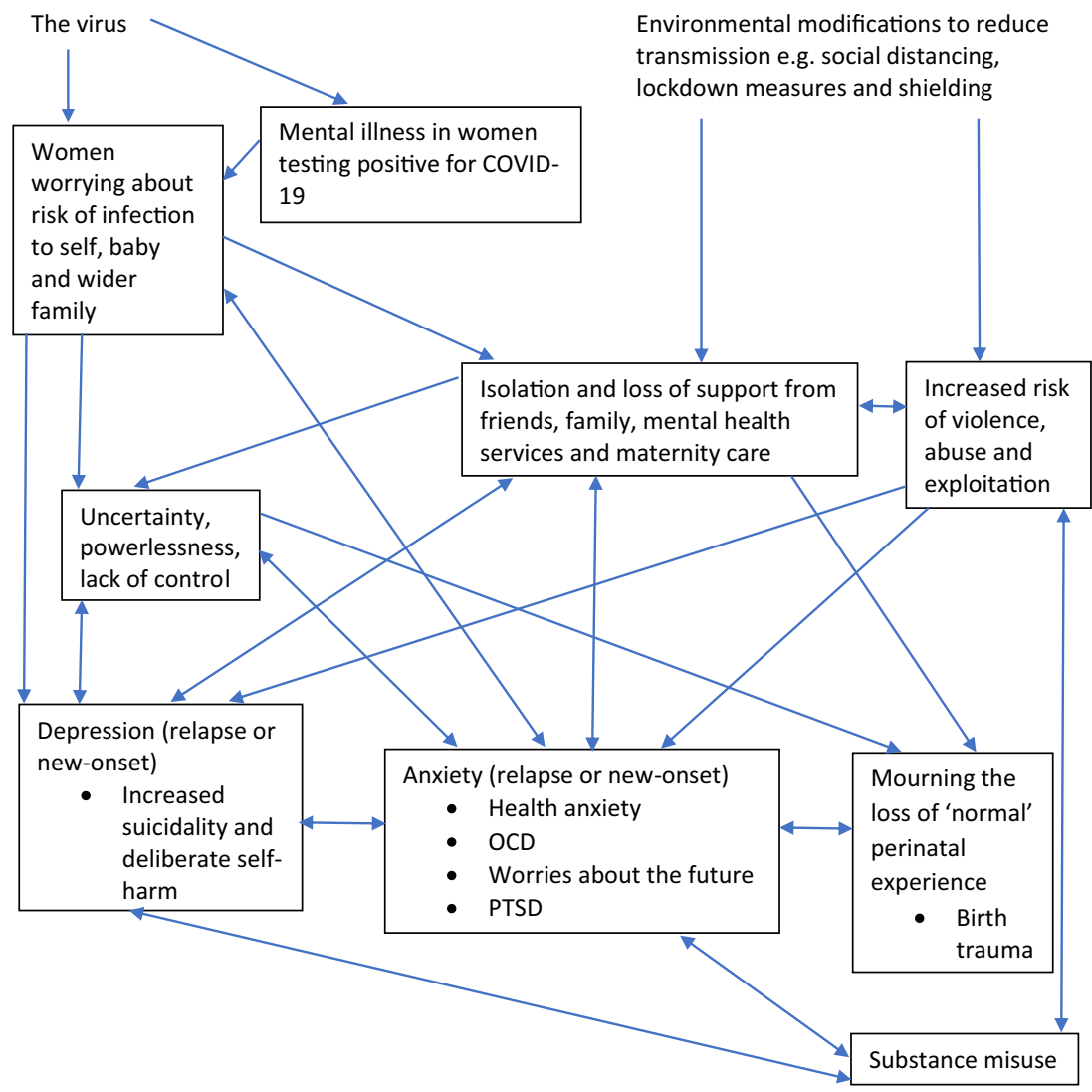

which some staff felt impacted on breastfeeding support: 'not getting hands on support'.

Infection control measures presented some specific challenges in the inpatient perinatal setting to the assessment of the mother-infant interaction. A few staff working on MBUs reflected on the challenges posed by the separation of women and babies with suspected COVID-19. Others were concerned about the impact of face mask wearing on the developing infant: 'babies ... cared for by masked nurses ... lack of interaction with facial features.'
Other open-ended questions elicited reflections on positive aspects of adaptations made to manage the impact which some staff felt would be beneficial to continue beyond the pandemic. Two themes of telemedicine benefits to service users and staff were identified. Several staff reflected on the flexibility of virtual appointments for women as busy new mothers and the range of online self-help and other supports available, such as cognitive behavioural therapy, mindfulness and third sector initiatives: 'local and national groups such as PANDAS stepping

Table 3 Challenges to perinatal work rated as very or extremely relevant by mental health staff

\begin{tabular}{|c|c|c|}
\hline & $n / N^{*}$ & $\%$ \\
\hline Challenges assessing mother and infant relationships because of lack of direct access & $90 / 208$ & 43.3 \\
\hline Difficulty planning and monitoring treatment due to reduced social care services & $67 / 209$ & 32.1 \\
\hline Difficulty planning and monitoring treatment due to reduced community midwife and health visitor services & $62 / 211$ & 29.4 \\
\hline Safeguarding procedures are more difficult than usual to mobilise & $62 / 211$ & 29.4 \\
\hline Reduced access to maternity units to carry out assessments & $59 / 211$ & 28.0 \\
\hline Referrals to our service not made or delayed because of the COVID-19 crisis & $58 / 210$ & 27.6 \\
\hline Reduced opportunities to admit to mother and baby units & $37 / 206$ & 18.0 \\
\hline Challenges arising from maternal or infant COVID-19 infection & $35 / 207$ & 16.9 \\
\hline $\begin{array}{l}\text { Children are too readily taken into care because of obstacles to making other assessment and management plans at the } \\
\text { present time }\end{array}$ & $9 / 208$ & 4.3 \\
\hline
\end{tabular}

${ }^{*}$ Total respondents for this item 
Reduced opportunities for assessment and support

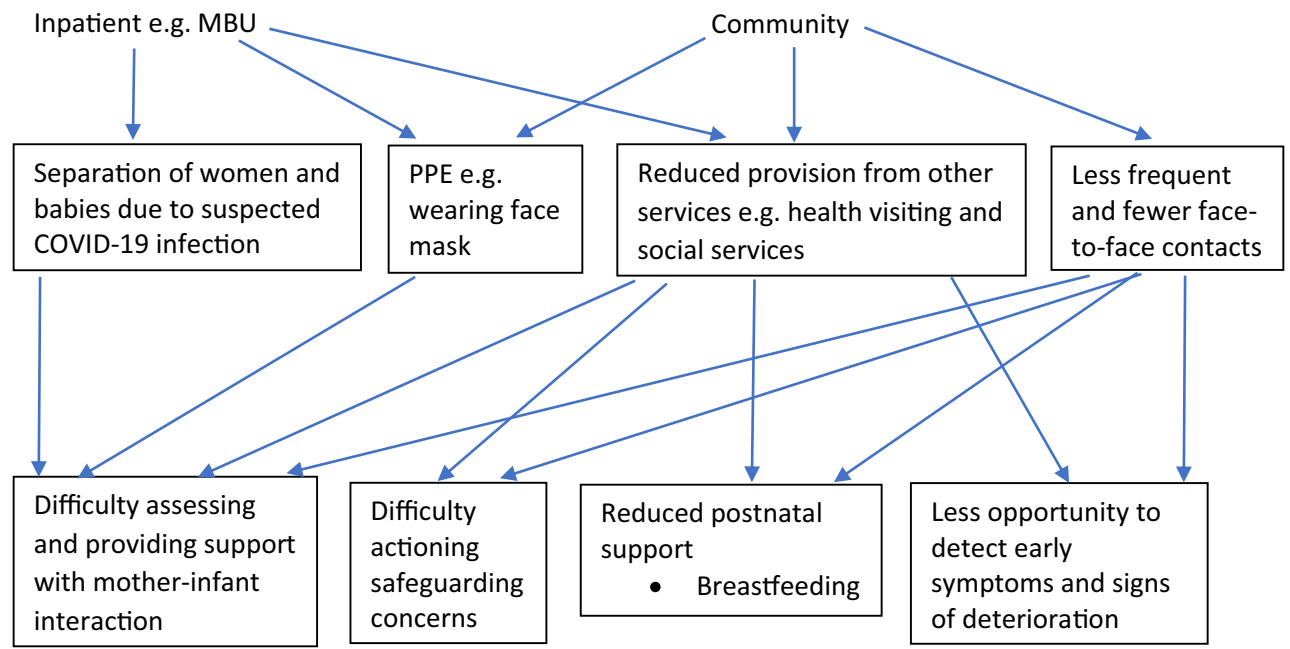

Fig. 2 Challenges to perinatal mental health service provision expressed by staff (themes in boxes)

up with online support.' Benefits to staff were related to time management; reduced travel when working from home resulted in increased time for clinical record keeping and improved work-life balance.

\section{Discussion}

\section{Main findings}

Mental health staff in this survey, administered during the COVID-19 pandemic lockdown in the UK, reported particular concerns about the mental health of women in the perinatal period. Staff who reported working with perinatal women more often endorsed as relevant for service users those items pertaining to social isolation and abusive relationships than in the full sample of mental health staff surveyed (Johnson et al. 2020). While there has been little published research on the impact of the pandemic on perinatal mental health, pregnant women in China (Liu et al. 2020; Wu et al. 2020) and Canada (Saccone et al. 2020) assessed following, versus those assessed prior to, the onset of the pandemic, reported greater levels of anxiety and depression symptoms. However, to our knowledge, there has been no study to date reporting on staff perceptions of mental health care for perinatal women.

Staff working in the perinatal setting were concerned about how to support women during pregnancy and in the postpartum period as a result of changes to care provided by their own and other services. Changes included reduced face-to-face contact in community mental health services, changes in infection control measures and reduced social care and home visiting services (i.e. maternity and health visiting services). Consequently, staff were worried about how to mobilise safeguarding procedures and assess and support the mother-infant interaction. There were also concerns about the impact of wearing masks on the developing infant; to our knowledge, there has been no research investigating the impact of this in residential settings such as MBUs. However, staff highlighted positive changes such as the greater flexibility provided by remote consulting.

The impact of COVID-19 infection on women and their infants was inevitably a concern. Neuropsychiatric complications of infection have been reported, including acute confusion (Rogers et al. 2020), which may mimic postpartum psychosis.

\section{Strengths and limitations}

This is the first study to document the experiences of mental health care staff supporting women in the perinatal period during the COVID-19 pandemic. The use of both qualitative and quantitative data facilitates the triangulation of our findings (Tracy 2010).

However, a limitation of surveys is that they may overrepresent those who are particularly distressed and keen to voice their concerns. Another limitation is that it was not possible to verify that respondents were definitely mental health professionals. There are also some limitations posed by the convenient nature of the sample. Despite efforts to recruit a broad ethnic demographic, the number of non-White respondents in this sample is relatively small. The sample is mainly female and there may be gendered attitudes which are overrepresented. There is also limited geographical diversity, with most of the respondents being from England; there may be different 
experiences in the UK's devolved nations, with differing levels of perinatal mental health service provision, which were not adequately captured. Most of the staff were working in community services, with only 18 respondents from the MBU. There were also no questions about the experiences of partners of perinatal women so these experiences were not captured.

Furthermore, the sample included both staff working only in perinatal services and other staff working with perinatal women among other service users or in nonperinatal settings. Yet, we believe the latter group is more representative of the structure of health services in many places outside the UK where there are no dedicated perinatal mental health services.

\section{Implications}

As has been expressed by others (Holmes et al. 2020; Johnson et al. 2020) and highlighted in this study, some changes to service delivery will suit some groups of service users more than others so support must continue to be individually tailored. Indeed our study highlighted challenges to remote consulting unique to the perinatal period. Face-to-face assessment is necessary in high-risk cases as highlighted by the recent confidential enquiry of maternal deaths in the UK during the first 3 months of the pandemic, which included four suicides and two domestic homicides (Knight et al. 2020).

However, the findings of this study have also highlighted some of the advantages of perinatal mental health care using virtual platforms. During and beyond the current pandemic, there is potential for the flexibility provided by remote consulting to reduce non-attendance rates (Rose et al. 2020). Despite this, interviews with women in maternity settings in the UK during the pandemic suggest that the use of virtual appointments made them reluctant to discuss mental health issues (Karavadra et al. 2020).

Future research would usefully focus on how to manage these particular areas of difficulty such as the assessment of perinatal mental health, DVA and the mother-infant relationship to equip staff with the tools to support women remotely and increase awareness of the circumstances under which remote assessment may be inappropriate. Further research to elucidate the direct effect of COVID19 infection on pregnant and breastfeeding women and the developing infant (Juan et al. 2020) would also help to address another major stressor for women during the pandemic. This would also allow perinatal mental health staff to provide women with accurate information about the risks posed by COVID-19 to reduce the uncertainty contributing to increased anxiety among the perinatal population during this time (Motrico et al. 2020).

\section{Conclusions}

In summary, there is an urgent need for research that provides an understanding of the experiences of perinatal women and their families during the COVID-19 pandemic, including potential barriers to supporting families during this time. Greater understanding could illuminate potential targets for interventions by mental health staff and other professionals to support women with their perinatal mental health and safeguard women and their families against violence and abuse during the current pandemic and beyond.

Lived experience commentary: Clare Dolman and Sarah Spring.

This survey highlights the challenges encountered by mental health professionals trying to deliver vital services during the pandemic but reading their views on what they thought had affected women with perinatal mental illness the most during the pandemic, we wondered what women themselves would say had been most relevant to them. I understand that another survey is being conducted to answer those very questions, but perhaps that's worth pointing out here.

There was no specific mention of the impact on fathers which one might expect when dealing with a perinatal population, though a couple of 'difficulties' related to families/carers. The results illustrate the detrimental effect of lack of face-to-face consultations, especially important to help women having problems with breastfeeding and bonding with their baby. Using video calls is referred to as a possible solution but they rely on a good connection, availability of the technology and a woman's ability to use such technology if cognitively challenged when struggling with depression or other mental illness so reliance on this medium may exclude many mothers. For those women with developing psychosis and other conditions involving hallucinations, contact other than face-to-face could exacerbate or precipitate more delusions and, as mothers with these illnesses are good at disguising their symptoms, the severity of their illness could go undetected. This is another reason why remote contact should be used with extreme caution.

In relation to the service difficulties identified during the pandemic, it is noteworthy that perinatal services weren't designated an essential service. This is an important point which should be considered in any future lockdown: many perinatal team staff were redeployed to hospital roles so women who had been considered serious enough to receive weekly therapeutic visits were only seen much less frequently thus increasing the risk that their condition would worsen. Similarly numbers of Health Visitors, who provide essential support to women in the perinatal period, were redeployed. This policy now appears short-sighted and in need of review.

This is an independently written perspective from lived experience contributed by some of the members of the IOPPN's Women's Mental Health Section's service user perinatal advisory group with relevant experience.

Acknowledgments We thank the clinicians who took the time during the pressures of the pandemic to complete the survey, including those who provided feedback on a pilot survey.

Author contributions S.J. drafted the survey and C.D.L. developed the online version. C.D.L. and A.S. led on recruitment strategies. S.J. and L.M.H. conceived the idea for the perinatal study. C.D.L. cleaned the data. C.A.W. designed the study analysis plan and analysed the 
data. Peer debriefing of qualitative data was provided by L.M.H. to C.A.W. C.A.W. wrote the first draft of the manuscript with input from L.M.H. All authors critically revised this draft and have approved the final version.

Funding This paper presents a secondary analysis of independent research commissioned and funded by the National Institute for Health Research (NIHR) Policy Research Programme, conducted by the NIHR Policy Research Unit (PRU) in Mental Health.

Data availability The survey dataset is currently being used for additional research by the author research group and is not currently available in a data repository. A copy of the survey is available at this web address: https://opinio.ucl.ac.uk/s?s=67819.

Code availability Not applicable.

\section{Declarations}

Ethics approval The King's College London research ethics committee approved this study (MRA-19/20-18372).

Consent to participate Information on participation was provided on the front page of the survey. By starting the survey, participants agreed that they had read and understood all of this information.

Consent for publication It was explained on the front page of the survey that responses may be used in articles published in scientific journals and that these articles would not include any information which could be used to identify any participant.

Conflict of interest S.J., S.O., L.M.H., and A.S. are grant holders for the NIHR Mental Health Policy Research Unit. L.M.H. receives salary support from the NIHR Biomedical Research Centre at South London and Maudsley NHS Foundation Trust and King's College London and the NIHR South London Applied Research Collaboration.

Disclaimer The views expressed are those of the authors and not necessarily those of the NIHR, the Department of Health and Social Care or its arm's length bodies or other government departments.

Open Access This article is licensed under a Creative Commons Attribution 4.0 International License, which permits use, sharing, adaptation, distribution and reproduction in any medium or format, as long as you give appropriate credit to the original author(s) and the source, provide a link to the Creative Commons licence, and indicate if changes were made. The images or other third party material in this article are included in the article's Creative Commons licence, unless indicated otherwise in a credit line to the material. If material is not included in the article's Creative Commons licence and your intended use is not permitted by statutory regulation or exceeds the permitted use, you will need to obtain permission directly from the copyright holder. To view a copy of this licence, visit http://creativecommons.org/licenses/by/4.0/.

\section{References}

Adhanom Ghebreyesus T (2020) Addressing mental health needs: an integral part of COVID-19 response. World Psychiatry 19:129130. https://doi.org/10.1002/wps.20768
Ayers S, Shakespeare J (2015) Should perinatal mental health be everyone's business? Prim Health Care Res Dev 16:323-325. https:// doi.org/10.1017/S1463423615000298

Braun V, Clarke V (2006) Using thematic analysis in psychology. Qual Res Psychol 3:77-101. https://doi.org/10.1191/1478088706qp063 oa

Caparros-Gonzalez RA, Alderdice F (2020) The COVID-19 pandemic and perinatal mental health. J Reprod Infant Psychol 38:223-225. https://doi.org/10.1080/02646838.2020.1786910

González-Sanguino C, Ausín B, Castellanos MÁ, Saiz J, López-Gómez A, Ugidos C, Muñoz M (2020) Mental health consequences during the initial stage of the 2020 Coronavirus pandemic (COVID19) in Spain. Brain Behav Immun 87:172-176. https://doi.org/10. 1016/j.bbi.2020.05.040

Holmes EA et al (2020) Multidisciplinary research priorities for the COVID-19 pandemic: a call for action for mental health science. Lancet Psychiatry 7:547-560

Hyland P et al (2020) Anxiety and depression in the Republic of Ireland during the COVID-19 pandemic. Acta Psychiatr Scand. https://doi.org/10.1111/acps.13219

Johnson $S$ et al (2020) Impact on mental health care and on mental health service users of the COVID-19 pandemic: a mixed methods survey of UK mental health care staff . Soc Psychiatry Psychiatr Epidemiol. https://doi.org/10.1007/s00127-020-01927-4

Juan J, Gil MM, Rong Z, Zhang Y, Yang H, Poon LC (2020) Effect of coronavirus disease 2019 (COVID-19) on maternal, perinatal and neonatal outcome: systematic review. Ultrasound Obstet Gynecol 56:15-27. https://doi.org/10.1002/uog.22088

Karavadra B, Stockl A, Prosser-Snelling E, Simpson P, Morris E (2020) Women's perceptions of COVID-19 and their healthcare experiences: a qualitative thematic analysis of a national survey of pregnant women in the United Kingdom. BMC Pregnancy Childbirth 20:600. https://doi.org/10.1186/s12884-020-03283-2

Knight M et al. (2020) Saving Lives, Improving Mothers' Care Rapid Report: Learning from SARS-CoV-2-related and associated maternal deaths in the UK March - May 2020 Oxford: National Perinatal Epidemiology Unit, University of Oxford. https://www.hqip.org.uk/wp-content/uploads/2020/08/Ref.201-MBRRACE-UK-maternal-COVID-19-Report-FINAL.pdf. Accessed 1 Sep 2020

Lincoln YS, Guba EG (1985) Naturalistic inquiry. Sage, Newbury Park. https://doi.org/10.1016/0147-1767(85)90062-8

Liu X et al. (2020) Prenatal anxiety and obstetric decisions among pregnant women in Wuhan and Chongqing during the COVID19 outbreak: a cross-sectional study. BJOG Int J Obstet Gynaecol 127:1229-1240. doi:https://doi.org/10.1111/1471-0528. 16381

Moreno C et al (2020) How mental health care should change as a consequence of the COVID-19 pandemic. Lancet Psychiatry 7(9):P813-824

Motrico E et al (2020) Good practices in perinatal mental health during the COVID-19 pandemic: a report from task-force RISEUP-PPD COVID-19. Clin Salud 31:155-160

Oram S, Khalifeh H, Howard LM (2017) Violence against women and mental health. Lancet Psychiatry 4:159-170. https://doi.org/10. 1016/S2215-0366(16)30261-9

Pierce $\mathrm{M}$ et al (2020) Mental health before and during the COVID-19 pandemic: a longitudinal probability sample survey of the UK population. Lancet Psychiatry. https://doi.org/10.1016/S22150366(20)30308-4

Rogers JP et al (2020) Psychiatric and neuropsychiatric presentations associated with severe coronavirus infections: a systematic review and meta-analysis with comparison to the COVID-19 pandemic. Lancet Psychiatry 7:611-627. https://doi.org/10.1016/S22150366(20)30203-0 
Rose E, Manoharan M, Powell J (2020) Impact of the COVID-19 pandemic on maternal mental health. BJPsych Advances:1-4. doi:https://doi.org/10.1192/bja.2020.78

Saccone $\mathrm{G}$ et al (2020) Psychological impact of coronavirus disease 2019 in pregnant women. Am J Obstet Gynecol 223:293-295. https://doi.org/10.1016/j.ajog.2020.05.003

Sheridan Rains L et al (2020) Early impacts of the COVID-19 pandemic on mental health care and on people with mental health conditions: framework synthesis of international experiences and responses. Soc Psychiatry Psychiatr Epidemiol. https://doi.org/10. 1007/s00127-020-01924-7

StataCorp L (2007) Stata data analysis and statistical Software Special Edition Release 10:733

Stein A et al (2014) Effects of perinatal mental disorders on the fetus and child. Lancet 384:1800-1819. https://doi.org/10.1016/S01406736(14)61277-0

The Lancet Psychiatry (2020) Mental health and COVID-19: change the conversation. Lancet Psychiatry 7:463
Tracy SJ (2010) Qualitative quality: eight “big-tent” criteria for excellent qualitative research. Qual Inq 16:837-851

UK Government (2020) Prime Minister's statement on coronavirus (COVID-19): 16 March 2020. https://www.gov.uk/government/ speeches/pm-statement-on-coronavirus-16-march-2020

Usher K, Bhullar N, Durkin J, Gyamfi N, Jackson D (2020) Family violence and COVID-19: Increased vulnerability and reduced options for support. Int J Ment Health Nurs 29:549-552

Wu Y et al (2020) Perinatal depressive and anxiety symptoms of pregnant women during the coronavirus disease 2019 outbreak in China. Am J Obstet Gynecol 223:240.e241-240.e249. https:// doi.org/10.1016/j.ajog.2020.05.009

Publisher's note Springer Nature remains neutral with regard to jurisdictional claims in published maps and institutional affiliations. 